\title{
Numerical investigation of fluid-structure interaction for long-span bridge decks
}

\author{
F. Gallerano, G. Cannata, L. Barsi \& S. Scarpone \\ Department of Civil, Construction and Environmental Engineering, \\ Sapienza University of Rome, Italy
}

\begin{abstract}
In this work a simulation model of aeroelastic phenomena for long-span bridges is presented. By the proposed model the aerodynamic field and the structural motion are simulated simultaneously and in a coupled manner. The structure is represented as a bidimensional rigid body with two degrees of freedom, having mass per unit length and mass moment of inertia per unit length equal to those of the deck. The aerodynamic fields are simulated by numerically integrating the Arbitrary Lagrangian-Eulerian (ALE) formulated Unsteady Reynolds-Averaged Navier-Stokes (URANS) equations with a finite volume scheme on moving grids which adapt themselves to the structural motion. The finite volume method is based on high order weighted essentially non-oscillatory (WENO) reconstructions. The time discretisation is performed by a five stage fourth order accurate strong stability preserving Runge-Kutta (SSPRK) method. The URANS equations are completed by the turbulent closure relations which are expressed as a function of the turbulent kinetic energy and the turbulent frequency according to the $k-\omega$ SST approach. The model validation is performed by the comparison between numerical and experimental results. The proposed model is utilised in order to identify the flutter critical wind velocity of the Forth Road Bridge deck, and the numerical results are compared with those of an experimental campaign. Keywords: fluid-structure interaction, bridge aeroelasticity, flutter.
\end{abstract}

\section{Introduction}

Long span bridges are susceptible to an oscillatory unstable aero-elastic phenomenon, named flutter, in which the bridge deck motion acquires divergent character and the oscillations amplitude grows rapidly up to causing the structural 
failure. Traditionally the critical flutter wind velocity of long span bridge decks is identified through the Scanlan approach. A central element of the abovementioned Scanlan approach lies in modelling the aerodynamic forces as linear functions of the structural displacements, under the assumption of purely sinusoidal motions. Astiz [1] highlights that the linear relation between the forces produced by the aerodynamic field on the deck and the structural displacements proves to be acceptable only in the event that the amplitude of structural oscillations is limited. An alternative approach, followed by different authors (Selvam et al. [2]; Robertson et al. [3]; Frandsen [4]; Braun and Awruch [5]) consists in simulating the aerodynamic fields and the structural motion simultaneously and in a coupled manner, so as to allow the identification of the critical flutter wind velocity in a direct way.

In this work a fluid-structure interaction model is presented in which the aerodynamic fields and the structural motion are simulated simultaneously and in a coupled manner. The aerodynamic fields are simulated by numerically integrating the Arbitrary Lagrangian-Eulerian (ALE) formulated Unsteady Reynolds Averaged Navier-Stokes (URANS) equations with a finite volume scheme on moving grids which adapt to the structural motion. The finite volume method is based on high order weighted essentially non-oscillatory (WENO) reconstructions. The time discretisation is performed by a five stage fourth order accurate strong stability preserving Runge-Kutta (SSPRK) method. The URANS equations are completed by the turbulent closure relations which are expressed as a function of the turbulent kinetic energy and the turbulence frequency according to the $k$ - $\omega$ SST turbulence model. In the proposed model the structure is represented as a bidimensional rigid body with two degrees of freedom, having mass per unit length and mass moment of inertia per unit length equal to those of the deck. The above-mentioned rigid body is attached to an elastic vertical spring and to an elastic torsional spring whose stiffnesses are calibrated in order to give the natural frequencies corresponding to the fundamental flexural and torsional natural modes of vibration of the structure. The model validation is performed by the comparison between numerical and experimental results related to the case study of the rectangular cylinder with aspect ratio equal to 1:5: this validation is performed by comparing the Strouhal number, the lift coefficient and the drag coefficient. As a further element of validation, the proposed model is applied to the case study of the Forth Road Bridge deck, and the results of the numerical simulations are compared with the experimental data of Robertson et al. [3] in terms of critical flutter wind velocity.

\section{The proposed model}

\subsection{The fluid motion equations}

The fluid dynamic field around the body is simulated by numerically integrating the 2D Unsteady Reynolds-Averaged Navier-Stokes (URANS) equations. Many authors simulate the fluid dynamic fields with finite volume techniques on unstructured grids (Casonato and Gallerano [6]; Oka and Ishihara [7]; Bruno and 
Khris [8]; Gallerano et al. [9]) and on structured grids (Rossmanith et al. [10]; Mannini et al. [11]; Gallerano and Cannata [12]; Haque et al. [13]). In the case in which the simulations of the fluid dynamic fields involve moving boundaries, the fluid motion equations have to be formulated according to the Arbitrary Lagrangian-Eulerian approach (ALE) (De Miranda et al. [14]; Nieto et al. [15]).

The ALE formulated ensemble-averaged continuity and momentum equations in integral form read as follows

$$
\begin{gathered}
\frac{d}{d t} \int_{\Delta A} d A+\int_{L}\left[\left\langle u_{i}\right\rangle-u_{g, i}\right] n_{i} d L=0 \\
\frac{d}{d t} \int_{\Delta A}\left\langle u_{i}\right\rangle d A=-\int_{L}\left\langle u_{i}\right\rangle\left[\left\langle u_{j}\right\rangle-u_{g, j}\right] n_{j} d L+\int_{\Delta A} \frac{\partial}{\partial x_{j}}\left[2 v\left\langle S_{i j}\right\rangle-\left\langle u_{i}^{\prime} u_{j}^{\prime}\right\rangle\right] d A \\
+\int_{\Delta A} f_{i} d A-\int_{\Delta A} \frac{\partial\langle P\rangle}{\partial x_{i}} d A
\end{gathered}
$$

where $d A$ is the area of a surface element whose contour line is $L, n_{j}$ is the outward normal, $\left\langle u_{i}\right\rangle$ and $\langle p\rangle$ are the ensemble-averaged $i$-th component of the fluid velocity and the ensemble-averaged fluid pressure, $u_{g, i}$ is the $\mathrm{i}$-th component of the grid velocity, $v$ is the kinematic viscosity and $f_{i}$ the $\mathrm{i}$-th component of the mass force vector. The unknown term $\left\langle u_{i}{ }^{\prime} u_{j}{ }^{\prime}\right\rangle$, which can be defined as the Reynolds tensor, is related to the ensemble-averaged strain rate tensor $\left\langle S_{i j}\right\rangle$ and the ensembleaveraged turbulent kinetic energy per unit mass $\langle k\rangle$ through the relation

$$
\left\langle u_{i}^{\prime} u_{j}^{\prime}\right\rangle=-2 v_{t}\left\langle S_{i j}\right\rangle+\frac{2}{3}\langle k\rangle \delta_{i j}
$$

where $v_{t}$ is the kinematic eddy viscosity and $\delta_{i j}$ is the Kronecker symbol. The turbulence closure relations and the calibration parameters which are included in them are derived from Menter [16].

\subsection{The structural motion equations}

The 2D motion of the body can be described in terms of two displacement components, $\eta, \theta$, where $\eta$ is the translational displacement component in the vertical direction $y$ (positive upwards), and $\theta$ denotes the rotational displacement component (positive nose-up). The governing equation for the body motion are

$$
\begin{aligned}
& m \ddot{\eta}+c_{\eta} \dot{\eta}+k_{y} \eta=f_{y} \\
& I \ddot{\theta}+c_{\theta} \dot{\theta}+k_{\theta} \theta=m_{\theta}
\end{aligned}
$$

where $m$ and $I$ are respectively the mass and the mass moment of inertia per unit length of the deck, $c_{y}$ and $c_{\theta}$ are respectively the structural damping coefficient in the vertical and torsional degree of freedom, $k_{y}$ and $k_{\theta}$ are respectively the stiffness constant of the vertical elastic spring and the stiffness constant of the torsional elastic spring, $f_{y}$ and $m_{\theta}$ are respectively the component in the $y$ direction of the 
above-mentioned force and the twisting moment $m_{\theta}$ generated by the same force on the body, and $\eta$ and $\theta$ are respectively the vertical displacement of the centre of gravity of the body and the rotational angle of the body around the shear centre. The component $f_{y}$ and the twisting moment $m_{\theta}$ are calculated by integrating the pressures, the viscous stresses and the turbulent stresses over the surface of the structure. The stiffness $k_{y}$ and $k_{\theta}$ are calibrated in order to give the natural frequencies corresponding to the fundamental flexural and torsional natural modes of vibration of the structure. The damping coefficients are calculated according to the formulation proposed in the work of Hines et al. [17] on the basis of the given damping ratios. The structural motion equations are solved by a second-order accurate scheme, and the coupling between the fluid solver and the structure solver follows a partitioned loose-coupling approach (Li et al. [18]).

\subsection{The numerical scheme}

The numerical method adopted in this work is the extension of the numerical method proposed by Gallerano et al. [19] for the integration of the 2D depthaveraged motion equations to the three-dimensional non-hydrostatic case by means of the finite volume method. Let us define $\left\langle\overline{u_{i}}\right\rangle$ and $\langle P\rangle$ as the cell averaged values of the velocity vector and the pressure

$$
\left\langle\bar{u}_{i}\right\rangle=\frac{1}{\Delta A} \int_{\Delta A}\left\langle u_{i}\right\rangle d A, \quad\langle\bar{P}\rangle=\frac{1}{\Delta A} \int_{\Delta A}\langle P\rangle d A
$$

The state of the system is known at the centre of the calculation cell and it is defined by the cell-averaged values $\left\langle\overline{u_{i}}\right\rangle$ and $\langle P\rangle$. $t^{(n)}$ is the time level of the known variables while $t^{(n+1)}$ is the time level of the unknown variables. From the values of the fluid dynamic quantities at the time $t^{(n)}$, by means of the structural motion equations, the structural displacements are calculated and, from the latter, the position of the cell vertices and the grid velocity $u_{g, i}{ }^{(n)}$ are calculated. Given at the time $t^{(n)}$ the values of $\left\langle\overline{u_{i}}\right\rangle^{(n)},\langle P\rangle^{(n)},\langle\bar{k}\rangle^{(n)},\langle\bar{\omega}\rangle^{(n)}$ at the centre of the calculation cells, the integration of the fluid motion equations (supplied with the turbulence closure relations for the Reynolds stress tensor) allows the calculation of $\left\langle\overline{u_{i}}\right\rangle{ }^{(n+1)}$, $\langle P\rangle^{(n+1)}$ at time $t^{(n+1)}$.

The solution procedure for the fluid motion equations uses a five stage fourth order accurate Strong Stability Preserving Runge-Kutta (SSPRK) fractional-step method for the momentum equations and applies a pressure correction formulation to obtain a divergence free velocity field at each time level. Let $\left\langle\overline{u_{i}}\right\rangle^{(n)}$ be the value of the $i$-th component of the fluid velocity at the time level $n$. The fluid velocity field $\left\langle\overline{u_{i}}\right\rangle^{(n+1)}$ at the time level $n+1$ is calculated through the following five stage iteration procedure. Let

$$
\left\langle\bar{u}_{i}\right\rangle^{(0)}=\left\langle\bar{u}_{i}\right\rangle^{(n)}
$$

At each stage $p$ (where $p=1,2, \ldots 5$ ) an auxiliary velocity field, $\left\langle\overline{u_{i}}\right\rangle^{*}(p)$, is obtained directly from eqn (2) using values from the previous time level 


$$
\left\langle\bar{u}_{i}\right\rangle^{*(p)}=\sum_{q=0}^{p-1}\left[\Omega_{p q}\left\langle\bar{u}_{i}\right\rangle^{(q)}+\Delta t \varphi_{p q} D\left[\left\langle\bar{u}_{i}\right\rangle^{(q)}, t^{(n)}+d_{q} \Delta t\right]\right]
$$

having indicated with $D\left(\left\langle u_{i}\right\rangle, t\right)$ the right-hand side of eqn (2) divided for $\Delta A$, in which the last term related to the pressure gradient has been omitted. See Spiteri and Ruuth [20] for the values of the coefficients $\Omega_{p q}, \varphi_{p q}$ and $d_{q}$. In general, the auxiliary velocity field of eqn (8) will not satisfy the continuity equation. As a result, the velocity and the pressure fields are corrected in the following manner. By introducing a scalar potential $\psi^{(p)}$ the well known Poisson pressure equation appears in the following integral form:

$$
\int_{L} \frac{\partial \psi^{(p)}}{\partial x_{i}} n_{i} d L=-\int_{L}\left\langle\bar{u}_{i}\right\rangle^{*(p)} n_{i} d L
$$

where $L$ and $n_{i}$ represent respectively the contour of the calculation cell and the $i$ th component of the outward unit vector normal to the contour. The solution of eqn (9) provides the calculation of the above-mentioned scalar potential $\psi^{(p)}$. The corrector velocity field $\left\langle\overline{u_{i}}\right\rangle^{c}$ is calculated through the following relation

$$
\left\langle\bar{u}_{i}\right\rangle^{c(p)}=\frac{\partial \psi^{(p)}}{\partial x_{i}}
$$

The calculation of the velocity at the stage $p$ is given by

$$
\left\langle\bar{u}_{i}\right\rangle^{(p)}=\left\langle\bar{u}_{i}\right\rangle^{*(p)}+\left\langle\bar{u}_{i}\right\rangle^{c(p)}
$$

The fluid velocity and pressure fields at the instant $t^{(n+1)}$ are respectively given by

$$
\left\langle\bar{u}_{i}\right\rangle^{(n+1)}=\left\langle\bar{u}_{i}\right\rangle^{(5)}, \quad\langle\bar{P}\rangle^{(n+1)}=\frac{1}{\Delta t} \psi^{(5)}
$$

For the calculation of term $D\left(\left\langle u_{j}\right\rangle, t\right)$ the numerical approximations of integrals on the right-hand side of eqn (2) is required. The aforementioned calculation is based on the following sequence:

1. High order WENO reconstructions, from cell averaged values, of the point values of the unknown variables at the center of the contour segments which define the calculation cells. At the center of the contour segment which is common with two adjacent cells, two point values of the unknown variables are reconstructed by means of two WENO reconstructions defined on two adjacent cells.

2. Advancing in time of the point values of the unknown variables at the center of the contour segments by means of the so-called exact solution of the Riemann problem, with initial data given by the pair of pointvalues computed by two WENO reconstructions defined on the two adjacent cells.

3. Calculation of the spatial integrals which define $D\left(\left\langle u_{j}\right\rangle, t\right)$. 
For further details on the WENO reconstructions, the advancing in time of the unknown variables and on the calculation of the spatial integrals which define $D\left(\left\langle u_{j}\right\rangle, t\right)$ (see Gallerano and Cannata [21] and Gallerano et al. [22]). The numerical integration of the turbulence closure relations allows the calculation of $\langle k\rangle^{(n+1)}$, $\langle\omega\rangle^{(n+1)}$ and the Reynolds stress tensor at the time $t^{(n+1)}$ via eqn (3). Discretising eqns (8) and (9) using the numerical method introduced above, entails the risk of introducing mass sources or sinks in the flow field if the velocity $u_{g, i}$ and the change volume over time are not treated consistently. For this reason, The Geometric Conservation Law (GCL)

$$
\frac{d}{d t} \int_{\Delta A} d A+\int_{\Delta A} \frac{\partial u_{g, i}}{\partial x_{i}} d A=0
$$

needs to be satisfied. To warrant consistency, eqn (13) is used to determine the grid velocity by the given change of volume of the computational cell (Hertel et al. [23]). In order to update the coordinates of the control volume vertices at all times, a mesh movement algorithm based on using Inverse Distance Weighting (Uyttersprot [24]) has been used in order to interpolate the displacements of the boundary nodes to the whole flow mesh.

\section{Model validation}

The model validation is performed by the comparison between numerical and experimental results. The aerodynamic fields that develop around a rectangular cylinder with aspect ratio $B / D$ equal to 5 (where $B=30 \mathrm{~cm}$ and $D=6 \mathrm{~cm}$ are respectively the width and the depth of the cross-section) are simulated. The simulations are performed in static conditions, i.e. all the degrees of freedom of the cross-section are restrained. The flow domain considered for the rectangular cylinder is $10 B$ by $5 B$. The total number of cells is 36800 . A constant velocity inlet has been set at the upwind boundary of the computational domain. At the solid walls the near-wall treatment proposed by Menter et al. [25] is used. The zero gradient boundary condition has been imposed at the outlet for all the fluid dynamic quantities (fluid velocity, turbulent kinetic energy, turbulence frequency).

For every performed simulation the time histories of drag and lift forces, and those of the fluid velocity at two different points placed in the wake of the body are determined. The time-averaged drag coefficient $C_{D}=F_{D} /\left(0.5 \rho U^{2} D\right)$ and the time-averaged lift coefficient $C_{L}=F_{L} /\left(0.5 \rho U^{2} B\right)$ (in which $F_{D}$ and $F_{L}$ are the drag and the lift forces exerted by the fluid on the structure, $U$ the undisturbed wind velocity, $D$ and $B$ the depth and the width of the deck, $\rho$ the fluid density) obtained numerically and those evaluated in the wind tunnel tests performed by Schewe $[26,27]$ are reported in table 1 . From table 1 it can be seen that the timeaveraged drag coefficients and the time-averaged lift coefficients calculated from the output data of the numerical simulations are very close to those obtained experimentally by the above-mentioned author. 
Table 1: Static coefficients (lift and drag) and Strouhal number of the rectangular cylinder from numerical simulations and experimental tests.

\begin{tabular}{|l|c|c|c|}
\hline & $\mathrm{C}_{\mathrm{D}}$ & $\mathrm{C}_{\mathrm{L}}$ & $\mathrm{St}$ \\
\hline Numerical (present work) & $1.092-1.194$ & 0 & $0.111-0.117$ \\
\hline Experimental (Schewe [26, 27]) & 1.029 & 0 & \\
\hline Experimental (Schewe [28]) & & & $0.111-0.115$ \\
\hline
\end{tabular}

The Strouhal number values obtained numerically and those obtained in the wind tunnel test performed by Schewe [28] are also reported in table 1. For every simulation performed, the Strouhal number is calculated as $S t=\left(f_{s} D\right) / U_{\infty}$, where the shedding frequency $f_{s}$ is computed by the time history of the fluid velocity at the two different points placed in the wake of the cylinder. From table 1 it can be seen that the Strouhal number values calculated by the output data of the numerical simulations range between values of 0.11 and 0.12 , in good agreement with the experimental data.

\section{Aeroelastic stability study of the Forth Road Bridge deck}

The proposed simulation model is used in order to evaluate the aero-elastic stability of the Forth Road Bridge deck. In table 2 the values of the geometrical parameters (maximum depth and overall width) and of the structural parameters (mass per unit length and mass moment of inertia per unit length, natural heaving and torsional frequency, heaving and torsional damping ratio) of the Forth Road Bridge deck are listed.

Table 2: $\quad$ Forth Road Bridge deck cross-sectional parameters.

\begin{tabular}{|l|l|}
\hline Depth & $3.2 \mathrm{~m}$ \\
\hline Width & $31.2 \mathrm{~m}$ \\
\hline Mass per unit of length & $17.3 \times 10^{3} \mathrm{~kg} / \mathrm{m}$ \\
\hline Moment of inertia per unit of length & $2.13 \times 10^{6} \mathrm{kgm} / \mathrm{m}$ \\
\hline Damping ratio (heaving natural mode of vibration) & $0.31 \%$ \\
\hline Damping ratio (torsional natural mode of vibration) & $0.14 \%$ \\
\hline Natural frequency (heaving natural mode of vibration) & $0.174 \mathrm{~Hz}$ \\
\hline Natural frequency (torsional natural mode of vibration) & $0.4 \mathrm{~Hz}$ \\
\hline
\end{tabular}

The flow domain considered for the bridge deck is $10 B$ by $5 B$. The total number of cells is 211000 . A constant velocity inlet has been set at the upwind boundary of the computational domain. At the solid walls the near-wall treatment proposed by Menter et al. [25] is used. The zero gradient boundary condition has been imposed at the outlet for all the fluid dynamic quantities (fluid velocity, turbulent kinetic energy, turbulence frequency). The numerical results are compared with those obtained from the wind tunnel tests described in the work of Robertson et al. [3]. It emerges from the simulations that the flutter critical wind velocity value 
is equal to $U^{*}=79.1 \mathrm{~m} / \mathrm{s}\left(U_{\theta}^{*}=U^{*} /\left(f_{\theta} B\right)=6.34\right.$, where $f_{\theta}$ is the natural torsional frequency of the deck), in good agreement with the experimental result of Robertson et al. $\left(U_{\theta^{*}} \approx 6.35\right)$. In agreement with that deduced by the same authors it is also found that, at the critical wind flutter velocity, the torsional oscillation frequency and the vertical oscillation frequency synchronize on a common value. This frequency value is equal to $0.34 \mathrm{~Hz}$.

In order to characterise the type of flutter instability which the Forth Road Bridge deck is prone to, consistently with the treatise of Matsumoto et al. [29] the angle $\Psi$ defined as the phase lag of the heaving response (vertical displacements) to the torsional response (rotations) of the structure is used. The above-mentioned authors highlight that the oscillatory motion of the cross-section of a bridge deck can be regarded as the superimposition of two fundamental oscillatory motions: the torsional fundamental mode and the heaving fundamental mode. The first one (torsional fundamental mode) is defined as a substantially torsional oscillatory motion accompanied by a vertical oscillatory motion of small entity. In the torsional fundamental mode, the phase angle $\Psi$ (as previously defined) is equal to $0^{\circ}$ or $180^{\circ}$ depending on whether the centre of rotation is placed upstream or downstream the mid-chord point of the deck cross-section. The second fundamental oscillatory motion (heaving fundamental mode) is defined as a substantially vertical oscillatory motion accompanied by a torsional oscillatory motion of small entity. In the heaving fundamental mode, the phase angle $\Psi$ (as previously defined) is equal to $90^{\circ}$ or $-90^{\circ}$ depending on whether the sign of the small rotation of the upward moving cross-section is clockwise or anti-clockwise. Bearing in mind the definition of the above-mentioned fundamental modes, Matsumoto et al. [29] characterise the type of coupled flutter of the cross-section of a bridge deck through the phase angle $\Psi$. Still consistently with the treatise of Matsumoto et al. [29], the torsional branch (TB) coupled flutter is defined as a coupled (torsional-flexural) flutter instability dominated by the fundamental torsional mode previously defined. In the case under examination (Forth Road Bridge deck), the relative contribution of the torsional fundamental mode to the instability of the structure is quantified as a value equal to $\cos \left(\Psi=-16^{\circ}\right)=0.96$ and the relative contribution of the heaving fundamental mode in a value equal to $-\operatorname{sen}\left(\Psi=-16^{\circ}\right)=0.27$. Therefore, it is concluded that the Forth Road Bridge deck is prone to a TB coupled flutter in which the torsional fundamental mode clearly dominates on the heaving fundamental mode.

In figs $1(\mathrm{a}), 1(\mathrm{~b}), 1(\mathrm{c}), 1(\mathrm{~d})$ the fluid velocity fields that develop around the deck in four instants within $1 / 2$ cycle of structural oscillations for an undisturbed wind velocity value of $87.4 \mathrm{~m} / \mathrm{s}\left(U_{\theta}=7.0\right)$ are shown. By $1 / 2$ cycle of structural oscillations is meant the temporal interval between the instant when the gravity centre of the downward moving structure corresponds to the static equilibrium position of the structure's centre of gravity and the instant when the gravity centre of the upward moving structure corresponds to the static equilibrium position of the structure's centre of gravity. In figs 2(a), 2(b), 2(c), 2(d) the distributions of the components normal to the deck surface of the forces per unit area exerted by the fluid on the structure in the same instants are shown. From the examination of figs $1(\mathrm{a}), 1(\mathrm{~b}), 1(\mathrm{c}), 1(\mathrm{~d})$ and figs 2(a), 2(b), 2(c), 2(d) it results that the reason for 

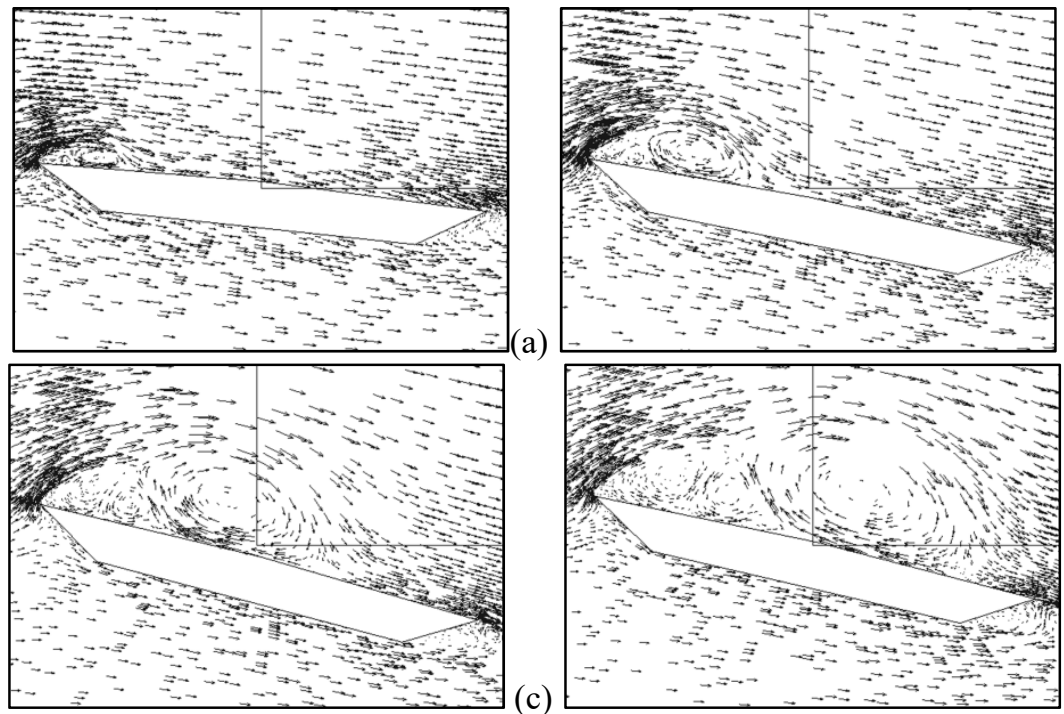

a)

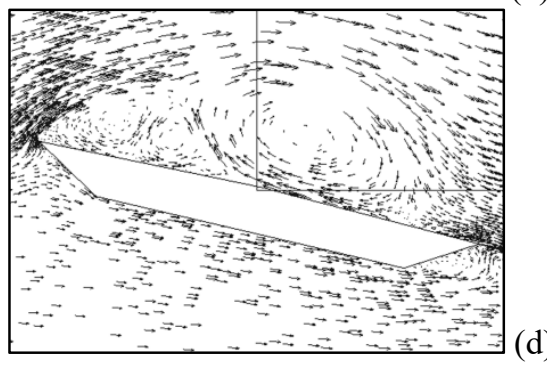

Figure 1: Fluid velocity fields around the Forth Road Bridge deck $\left(U_{\theta}=7.0\right)$.

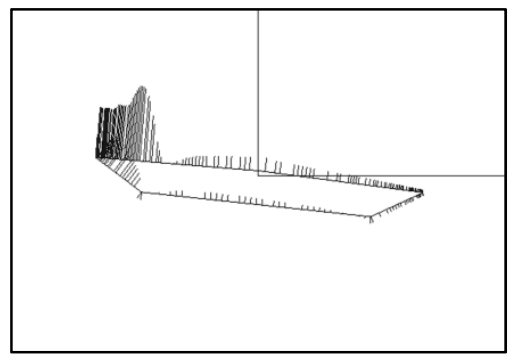

(a)

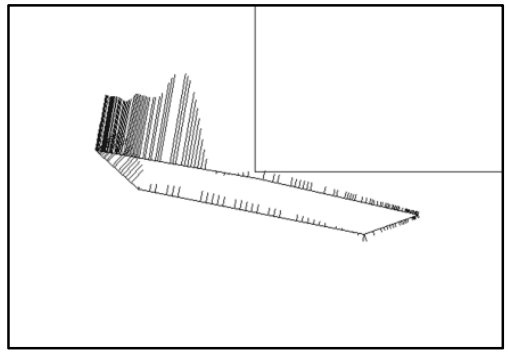

(b)
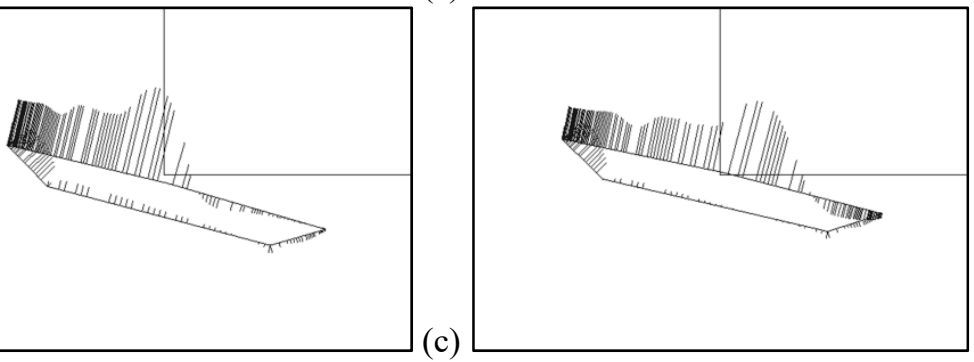

(d)

Figure 2: $\quad$ Pressure distributions around the Forth Road Bridge deck $\left(U_{\theta}=7.0\right)$.

the torsional-branch coupled flutter instability lies in the formation and the drift of large vortical formations along the deck surface. In particular, from the simulation of the phenomenon it is possible to deduce that the resultant of the vertical components of the forces per unit area exerted by the fluid on the deck surface moves with the vortical formation generated at the leading edge of the deck cross- 
section. The point of application of this resultant is placed at the vortical formation. The movement of the same resultant with respect to the shear centre of the deck cross-section gives rise to twisting moments varying in intensity and direction during the oscillation of the deck. Basing on these considerations it emerges that, during the whole $1 / 2$ cycle of structural oscillations (as previously defined), the sign of the twisting moment generated by the resultant of the components normal to the upper surface of the forces acting on the structure is always coherent with the sign of rotation. Consequently, there is a continuous supply of energy from the fluid dynamic field to the structure, that constitutes the reason for the instability of the torsional motion.

\section{Concluding remarks}

In this work a numerical investigation of aeroelastic phenomena for long-span bridges has been presented. With the proposed model the aerodynamic field and the motion of structure have been simulated simultaneously and in a coupled manner. The aerodynamic fields have been simulated by numerically integrating the Arbitrary Lagrangian-Eulerian (ALE) formulated Unsteady ReynoldsAveraged Navier-Stokes (URANS) equations with a finite volume scheme on moving grids which adapt to the structural motion.

The numerical model has been validated by the comparison between numerical and experimental results, and has been utilised for studying the aeroelastic stability of the Forth Road Bridge deck. It has been demonstrated that the reason for the torsional-branch coupled flutter instability of the Forth Road Bridge deck lies in the formation and the drift of large vortical formations along the deck surface. The sign of the twisting moment is always coherent with the sign of rotation, and there is a continuous supply of energy from the fluid dynamic field to the structure that constitutes the reason of the instability of the torsional motion.

\section{References}

[1] Astiz, M. A., Flutter stability of Very Long Suspension Bridges. Journal of Bridge Engineering, 3(3), pp. 132-139, 1998.

[2] Selvam, R.P., Govindaswamy, S. \& Bosch, H., Aeroelastic analysis of bridges using FEM and moving grids. Wind and Structures, 5(2-3-4), pp. 257-266, 2002.

[3] Robertson, I., Sherwin, S. J. \& Bearman, P. W., Flutter instability prediction techniques for bridge deck sections. International Journal for Numerical Methods in Fluids, 43(10-11), pp. 1239-1256, 2003.

[4] Frandsen, J.B., Numerical bridge deck studies using finite elements. Part I: flutter. Journal of Fluids and Structures, 19(2), pp. 171-191, 2004.

[5] Braun, A. L. \& Awruch, A. M., Finite element simulation of the wind action over bridge sectional models: Application to the Guamà River Bridge (Parà State, Brazil). Finite Elements in Analysis and Design, 44(3), pp. 105-122, 2008. 
[6] Casonato, M. \& Gallerano, F., A finite difference self-adaptive mesh solution of flow in a sedimentation tank. International Journal for Numerical Methods in Fluids, 10(6), pp. 697-711, 1990.

[7] Oka, S. \& Ishihara, T., Numerical study of aerodynamic characteristics of a square prism in a uniform flow. Journal of Wind Engineering and Industrial Aerodynamics, 97(11-12), pp. 548-559, 2009.

[8] Bruno, L. \& Khris, S., The validity of 2D numerical simulations of vortical structures around a bridge deck. Mathematical and Computer Modelling, 37(7-8), pp. 795-828, 2003.

[9] Gallerano, F., Pasero, E. \& Cannata, G., A dynamic two-equation Sub Grid Scale model. Continuum Mechanics and Thermodynamics, 17(2), pp. 101123, 2005.

[10] Rossmanith, J. A., Bale, D. S. \& LeVeque, R. J. A wave propagation algorithm for hyperbolic systems on curved manifolds. Journal of Computational Physics, 199(2), pp. 631-662, 2004.

[11] Mannini, C., Soda, A., Voß, R. \& Schewe, G., Unsteady RANS simulation of flow around a bridge section. Journal of Wind Engineering and Industrial Aerodynamics, 98(12), pp. 742-753, 2010.

[12] Gallerano, F. \& Cannata, G., Compatibility between reservoir sediment flushing and river protection. Journal of Hydraulic Engineering, 137(10), pp. 1111-1125, 2011.

[13] Haque, Md. N., Katsuchi, H., Yamada, H. \& Nishio, M., Flow field analysis of a pentagonal-shaped bridge deck by unsteady RANS. Engineering Applications of Computational Fluid Mechanics, 10(1), pp. 1-16, 2015.

[14] De Miranda, S., Patruno, L., Ubertini, F. \& Vairo, G., On the identification of flutter derivatives of bridge decks via RANS turbulence models: Benchmarking on rectangular prisms. Engineering Structures, 76, pp. 359370, 2014.

[15] Nieto, F., Hargreaves, D. M., Owen, J. S. \& Hernandez, S., On the applicability of 2D URANS and SST $k$ - $\omega$ turbulence model to the fluidstructure interaction of rectangular cylinders. Engineering Applications of Computational Fluid Mechanics, 9(1), pp. 157-173, 2015.

[16] Menter, F. R., Review of the shear-stress transport turbulence model experience from an industrial perspective. International Journal of Computational Fluid Dynamics, 23(4), pp. 305-316, 2009.

[17] Hines, J., Thompson, G. P. \& Lien, F. S., A turbulent flow over a square cylinder with prescribed and autonomous motions. Engineering Applications of Computational Fluid Mechanics, 3(4), pp. 573-586, 2009.

[18] Li, L., Sherwin, S. J. \& Bearman, P. W., A moving frame of reference algorithm for fluid/structure interaction of rotating and translating bodies. International Journal for Numerical Methods in Fluids, 38(2), pp. 187-206, 2002.

[19] Gallerano, F., Cannata, G. \& Tamburrino, M., Upwind WENO scheme for Shallow Water Equations in contravariant formulation. Computer \& Fluids, 62, pp. 1-12, 2012. 
[20] Spiteri, R. J. \& Ruuth, S. J., A new class of optimal high-order strongstability preserving time discretization methods. SIAM Journal on Numerical Analysis, 40(2), pp. 469-91, 2002.

[21] Gallerano, F. \& Cannata, G., Central WENO scheme for the integral form of contravariant shallow-water equations. International Journal for Numerical Methods in Fluids, 67(8), pp. 939-959, 2011.

[22] Gallerano, F., Cannata, G. \& Villani, M., An integral contravariant formulation of the fully non-linear Boussinesq equations. Coastal Engineering, 83, pp. 119-136, 2014.

[23] Hertel, C., Schumichen, M., Lobig, S., Frohlich, J. \& Lang, J., Adaptive large eddy simulation with moving grids. Theoretical and Computational Fluid Dynamics, 27, pp. 817-841, 2013.

[24] Uyttersprot, L., Inverse Distance Weighting Mesh Deformation. Master of Science Thesis, Delft University of Technology, 2014.

[25] Menter, F., Carregal Ferreira, J., Esch, T. \& Konno, B., The SST Turbulence Model with Improved Wall Treatment for Heat Transfer Predictions in Gas turbines. Proc. of the Int. Gas Turbine Cong.: Tokyo, pp. 1-7, 2003.

[26] Schewe, G., Influence of the Reynolds-number on flow-induced vibrations of generic bridge sections. Proc. of the Int. Conf. on Bridges: Dubrovnik, pp. 351-358, 2006.

[27] Schewe, G., Reynolds-number effects in flow around a rectangular cylinder with aspect ratio 1:5. Proc. of the Fifth European and African Conf. on Wind Engineering: Florence, 2009.

[28] Schewe, G., Reynolds-number-effects in flow around a rectangular cylinder with aspect ratio 1:5. Journal of Fluids and Structures, 39, pp. 15-26, 2013.

[29] Matsumoto, M., Matsumiya, H., Fujiwara, S. \& Ito, Y., New consideration on flutter properties based on step-by-step analysis. Journal of Wind Engineering and Industrial Aerodynamics, 98(12), pp. 429-437, 2010. 